\title{
Computational genome analyses of metabolic enzymes in Mycobacterium leprae for drug target identification
}

\author{
Anusuya Shanmugam ${ }^{1}$, Jeyakumar Natarajan ${ }^{2} *$ \\ ${ }^{1}$ Department of Bioinformatics, VMKV Engineering College, Vinayaka Missions University, Salem; ${ }^{2}$ Department of Bioinformatics, \\ Bharathiar University, Coimbatore; Jeyakumar Natarajan - Email: n.jeyakumar@yahoo.co.in; *corresponding author.
}

Received November 04, 2009; accepted March 06, 2010; published March 31, 2010

\begin{abstract}
:
Leprosy is an infectious disease caused by Mycobacterium leprae. M. leprae has undergone a major reductive evolution leaving a minimal set of functional genes for survival. It remains non-cultivable. As M. leprae develops resistance against most of the drugs, novel drug targets are required in order to design new drugs. As most of the essential genes mediate several biosynthetic and metabolic pathways, the pathway predictions can predict essential genes. We used comparative genome analysis of metabolic enzymes in M. leprae and H. sapiens using KEGG pathway database and identified 179 non-homologues enzymes. On further comparison of these 179 non-homologous enzymes to the list of minimal set of 48 essential genes required for cell-wall biosynthesis of $M$. leprae reveals eight common enzymes. Interestingly, six of these eight common enzymes map to that of peptidoglycan biosynthesis and they all belong to Mur enzymes. The machinery for peptidoglycan biosynthesis is a rich source of crucial targets for antibacterial chemotherapy and thus targeting these enzymes is a step towards facilitating the search for new antibiotics.
\end{abstract}

Keywords: Comparative genomics, Mur enzymes, M. leprae, Leprosy.

Background:

The availability of the complete genome sequences of several pathogenic bacteria and the completion of the human genome project has revolutionized the field of drug-discovery against threatening human pathogens [1]. Novel drug targets are required in order to design new drugs against antibiotic sensitive pathogens. In general, a target should provide adequate selectivity yielding a drug which is specific or highly selective against the pathogen with respect to the human host. Moreover, the target should be essential for growth and viability of the pathogen at least under the condition of infection [2]. The search for potential drug targets has increasingly relied on genomic approaches. The entire approach is built on the assumption that the potential target must play an essential role in the pathogen's survival and constitute a critical component in its metabolic pathway. At the same time, this target should not have any well-conserved homolog in the human host. This would preclude the possibilities of unacceptable crossreactivity that might prove detrimental to the host [3]. Leprosy is caused by Mycobacterium leprae, which primarily affects the skin, mucous membranes and peripheral nerves causing deformities. Leprosy remains a major global health problem, especially in the developing world. For over a century leprosy has presented major challenges in the fields of microbiology, pathology, immunology, and genetics; it continues to do so today. Computer analysis demonstrated that only half of the sequence contains protein-coding genes. The other half contains pseudo genes and non-coding sequences. These findings indicate that $M$. leprae has undergone a major reductive evolution leaving a minimal set of functional genes for survival [4]. Study of the coding region of the sequence provides evidence accounting for the particular pathogenic properties of $M$. leprae which is an obligate intracellular parasite. Mycobacterium leprae remains non-cultivable [5]. $M$. leprae had mutational changes in some of their genes like gyrA, rpoB, and folP which developed resistance against drugs like newer quinolones, refampicin and dapsone [6]. Resistant strains of $M$. leprae appeared due to mutations in the macrolide target, the ribosome [7]. These findings suggest the emergence of multi-drug resistant $M$. leprae. Hence the mycobacterial cell wall with its specific composition and structure is considered to be a major factor in promoting the natural resistance of mycobacteria to various antibiotics. Early detection of Mycobacterium leprae infection is considered an important component of strategies aiming at reducing transmission of infection, but currently available diagnostic tools often lack sufficient sensitivity and specificity to reach this goal [8]. In clinical studies, notable progress has been made concerning the immunology and immunopathology of leprosy, the genetics of human resistance, mechanisms of nerve injury, and chemotherapy. In nearly all of these areas, however, leprosy remains poorly understood compared to other major bacterial diseases [9]. Here, we present a computational approach to identify the genes essential to $M$. leprae using comparative pathway analysis followed by mapping of non-homologues genes with list of minimal set of essential genes required for cell-wall biosynthesis of $M$. leprae. In addition, our approach successfully identified a unique group of common enzymes as promising protein targets for new antibiotic development and further characterization in the laboratory.

Methodology:

Collection of metabolic pathway enzymes of $M$. leprae

Kyoto Encyclopedia of Genes and Genomes (KEGG) [10] is a collection of online databases dealing with genomes, enzymatic pathways, and biological chemicals. KEGG maintains five main databases. They are KEGG Atlas, KEGG Pathway, KEGG Genes, KEGG Ligand and KEGG BRITE. First, we collected all the metabolic pathways of $M$. leprae and $H$. sapiens from KEGG pathway database. Each of the pathways of $M$. leprae was compared with all the available pathways of $H$. sapiens to identify whether that particular pathway of $M$. leprae is present in $H$. sapiens or not. The pathways which were present in both $M$. leprae and $H$. sapiens were separated out and were named as shared pathways. The pathways which were present only in $M$. leprae but were not present in $H$. sapiens were grouped together and were called as unique pathways. The gene name and the enzyme commission number (EC) of all the enzymes present in both shared and unique pathways were identified and collected from KEGG Genes database.

\section{Retrieval of protein sequences and BLAST}

The protein sequence of all enzymes in both shared and unique pathways of $m$. leprae were retrieved from UNIPROT [11] in FASTA format. Each protein sequence was subjected to BLASTP analysis against the $H$. sapiens at an E-value cutoff of $10^{-4}$ [12]. BLAST results with no hits with $H$. sapiens were identified as nonhomologues enzymes of $M$. lepare.

\section{Identification of essential Enzymes}

The minimal set of essential genes required for cell envelope biosynthesis of $m$. leprae was reported previously using comparative genome sequence method by Vissa and Brennan [13]. The $M$. leprae enzymes which were non homologous to $H$. sapiens were mapped with the gene list of Vissa \& Brennan and the most common M. leprae genes were identified and further explored.

\section{Results and discussion:}

\section{Metabolic pathway information}

In KEGG pathway database we found 99 metabolic pathways for $M$. leprae and 210 metabolic pathways for $H$. sapiens. Out of 99 metabolic pathways for $M$. leprae five pathways are unique to $M$. leprae alone and comprised of 29 enzymes and remaining 94 
pathways are present in $H$. sapiens as well and comprised of 731 enzymes. The five unique pathways of $m$. leprae, 29 enzymes specific to these pathways and their corresponding gene id and the EC number were represented Table 1 (see supplementary material).

\section{Prediction of enzymes which were non homologous to human} Removing enzymes from the pathogen that share a similarity with the host protein ensures that the targets have nothing in common with the host proteins and thereby, eliminating undesired host protein-drug interactions. BLASTP similarity search of all these 760 (29 unique +731 shared) enzymes at an $E$-value cutoff of $10^{-4}$ resulted 179 non-homologues enzymes of $m$. leprae of which ten enzymes from the unique pathways and the remaining 169 belong to enzymes from shared pathways. All these 179 enzymes with their corresponding gene-id and EC number were represented in Table 2 (see supplementary material).

Comparison of non-homologues enzymes with essential gene set The $179(10+169)$ non-homologues enzymes were further compared to the minimal set of 48 essential genes required for cellwall biosynthesis of $M$. leprae and reported by Vissa and Brennan [13]. There are eight enzymes common in both data sets (Table 3 in supplementary material). Among the eight common enzymes only one enzyme was found to be present in unique pathway and the remaining seven enzymes were found to be present in shared pathways. All these eight enzymes were categorized as essential enzymes of $m$. leprae.

\section{Role of essential enzymes of $M$. leprae}

All the eight essential enzymes were further analyzed for the identification of potential drug targets. One of the eight essential enzymes Alanine racemase (alr) is the enzyme found in D-Alanine metabolism which is a unique pathway of M. leprae. It is also found in Alanine and Aspartate metabolism which is a shared pathway of $M$. leprae. Another essential enzyme Putative dTDP-4dehydrorhamnose 3, 5-epimerase ( $\mathrm{rmlC})$ was found to be essential for Nucleotide sugar metabolism which is a shared pathway of $M$. leprae. It was also found to be essential for polyketide sugar unit biosynthesis which is a unique pathway of M. leprae. The remaining 6 essential enzymes murC, murD, murE, murF, murG and murY were found to be essential for Peptidoglycan biosynthesis. It is noteworthy that all these 6 enzymes belong to the same family. This particular pathway, peptidoglycan biosynthesis was analyzed for the prediction of drug targets.

\section{Peptidoglycan biosynthesis and Mur enzymes}

M. leprae posses a multilayered cell envelope which basically consisted of, from inner to outer layer, a plasma membrane (PM), a peptidoglycan layer (PG), an electron translucent layer (ETL), and an irregular electron dense outer layer $(\mathrm{OL})$ [14]. This bacterial cell envelope provides strength and rigidity to counteract internal osmotic pressure, and protection against the environment. The peptidoglycan layer gives the cell wall its strength, and helps to maintain the overall shape of the cell. The basic peptidoglycan structure of both Gram-positive and Gram-negative bacteria is comprised of a sheet of glycan chains connected by short crosslinking polypeptides. Biosynthesis of peptidoglycan is a multi-step process comprising three main stages: (1) Formation of UDP-Nacetylmuramic acid (UDPMurNAc) from $\mathrm{N}$-acetylglucosamine (GlcNAc). (2) Addition of a short polypeptide chain to the UDPMurNAc. (3) Addition of a second N-acetylglucosamine (GlcNAc) to the disaccharide-pentapeptide building block and transport of this unit through the cytoplasmic membrane and incorporation into the growing peptidoglycan layer.

The second step of the petidoglycan biosynthesis was carried out by four of the mur ligase enzymes MurC, MurD, MurE and MurF. These four Mur ligases are responsible for the successive additions of L-alanine, D-glutamate, meso-diaminopimelate or L-lysine, and
D-alanyl-D-alanine to UDP-N-acetylmuramic acid. The final step in the formation of peptidoglycan was carried out by murG enzyme (N-acetyl glucosaminyl transferase). This enzyme is peripherally associated with the inner face of the cytoplasmic membrane. Therefore, the peptidoglycan subunit is completely assembled before it traverses the cytoplasmic membrane. Phospho-Nacetylmuramoyl-pentapeptide-transferase (mraY) is an important enzyme in murein synthesis. It is responsible for the formation of the first lipid intermediate of the cell wall peptidoglycan synthesis [15]. As the layer of the bacterial cell wall that confers strength is the peptidoglycan meshwork if we target murC, murD, murE and murF which catalyze the addition of a short polypeptide chain to the UDP-N-acetylmuramic acid (UDPMurNAc), we can easily prevent the synthesis of bacterial cell wall. Thus, these are excellent candidates for further exploration.

\section{Conclusion:}

The availability of full genome sequences and computer-aided analysis to identify probable antimicrobial drug targets has become a new trend in pharmacogenomics. The use of a comprehensive set of unique pathways and enzymes present in these pathways of $M$. leprae to identify new drug targets were documented in this study. We have found peptidoglycan biosynthetic pathway and the six mur enzymes (murC, murD, murE, murF, murG and murY) involved in this pathway to be used as potential drug targets. Protein structure and inhibitors of these important enzymes are not currently available. Further analysis on the structural studies on these mur enzymes is believed to provide valuable insights towards the design of an inhibitor specific to the peptidoglycan biosynthesis of $M$. leprae for the treatment of leprosy. The availability of the newer anti-leprotic drugs in the future would definitely support our present findings such that there would be a possibility of mur enzymes which were proposed by us for targeting M. leprae.

\section{Acknowledgment:}

We would like to acknowledge Dr. R. Ravishankar and Dr. S. Sivamani for critical reading of the manuscript and for helpful discussions.

\section{References:}

[1] L Miesel et al., Nat. Rev. Genet. (2003) 4: 442 [PMID:12776214]

[2] MK Sakharkar et al., In Silico Biol. (2004) 4: 0028 [PMID:15724285]

[3] B Frtiz, GA Raczniak, BioDrugs. (2002) 16: 331 [PMID:12408737]

[4] N Honore, Med Trop (Mars) (2002) 62: 473 [PMID:12616936]

[5] DM Scollard et al., Clin Microbiol Rev (2006) 19: 338 [PMID:16614253]

[6] S Maeda, Antimicrob Agents Chemother. (2001) 45: 3635 [PMID:11709358]

[7] F Doucet-Populaire et al., Curr Drug Targets infect Disord 2002 Dec; 2(4): 355-70 [PMID:12570741]

[8] A Geluk et al., Infect Immun. 2005 Sep; 73(9): 5636-44 [PMID:16113281]

[9] DM Scollard et al., Clin Microbiol Rev. 2006 Apr; 19(2): 33881 [PMID:16614253]

[10] http:// www.genome.jp/kegg.html

[11] http:// www.uniprot.org/

[12] Altschul et al., Nucleic Acids Res. 1997 Sep, 1:25(17): 33893402 [PMID:9254694]

[13] VD Varalakshmi, PJ Brennan, Genome Biology (2001) 2(8):Reviews1023 [PMID: 11532219]

[14] AI Takade et al., Microbiol Immun. (2003) 47(4): 265-70 [PMID:12801063]

[15] http://www.ebi.ac.uk/interpro

Edited by P. Kangueane Citation: Shanmugam \& Natarajan, Bioinformation 4(9): 392-395 (2010)

License statement: This is an open-access article, which permits unrestricted use, distribution, and reproduction in any medium, for noncommercial purposes, provided the original author and source are credited. 


\section{Supplementary material:}

Table 1: The five unique pathways and 29 enzymes specific to these pathways of $m$. leprae

\begin{tabular}{|c|c|c|c|}
\hline S. No & Name of the Pathway & Enzyme & EC \# \\
\hline \multicolumn{4}{|c|}{ 1. D-Alanine metabolism } \\
\hline 1 & D-Alanyl-alanine synthetase A & Ddl & 6.3.2.4 \\
\hline 2 & Alanine racemase & Alr & 5.1.1.1 \\
\hline \multicolumn{4}{|c|}{ 2. Ethylbenzene degradation } \\
\hline 3 & Acetyl-CoA acyltransferase & fadA & 2.3.1.16 \\
\hline 4 & Mycolytransferase & fbpA & 2.3.1.- \\
\hline \multicolumn{4}{|c|}{ 3. Benzoate degradation via CoA ligation } \\
\hline 5 & Monooxygenase & & 1.14.13.- \\
\hline 6 & Hypothetical protein & & 2.7.1.- \\
\hline 7 & Amidase & amiE & 3.5.1.4 \\
\hline 8 & Antigen 85-A & $\mathrm{fbpA}$ & 2.3.1.- \\
\hline 9 & Putative acyl-CoA synthetase & fadD32 & 6.2.1.- \\
\hline 10 & Enoyl-CoA hydratase & echA1 & 4.2.1.17 \\
\hline 11 & Acetyl-CoA acetyltransferase & fadA4 & 2.3.1.9 \\
\hline 12 & Succinate dehydrogenase iron-sulfur subuni & $\operatorname{sdh} B$ & 1.3.99.1 \\
\hline \multicolumn{4}{|c|}{ 4. 1,1,1-Trichloro-2,2-bis(4-chlorophenyl)ethane (DDT) degradation } \\
\hline 13 & Trans-acting enoyl reductase & & 1.3.1.- \\
\hline 14 & Putative oxidoreductase & & $1.2 .-.-$ \\
\hline \multicolumn{4}{|c|}{ 5. Two component system } \\
\hline 15 & Acetyl-CoA acetyltransferase & A to $\mathrm{B}$ & 2.3.1.9 \\
\hline 16 & cytochrome $\mathrm{c}$ oxidase subunit $\mathrm{XV}$ assembly protein (A) & CtaA & \\
\hline 17 & Glutamine synthetase & $\mathrm{G} \ln \mathrm{A}$ & 6.3 .1 .2 \\
\hline 18 & Putative two-component response regulator & MprA & \\
\hline 19 & Putative two-component system sensor histidine kinase & MprB & 2.7 .13 .3 \\
\hline 20 & Putative two-component response regulator & MtrA & \\
\hline 21 & Putative two-component system sensor histidine kinase & MtrB & 2.7.13.3 \\
\hline 22 & Putative serine protease & PepD & 3.4.21.- \\
\hline 23 & Two-component response regulator & PrrA & \\
\hline 24 & Sensor histidine kinase & PrrB & 2.7.13.3 \\
\hline 25 & Tryptophan synthase subunit alpha & $\operatorname{TrpA}$ & 4.2.1.20 \\
\hline 26 & Tryptophan synthase subunit beta & TrpB & 4.2.1.20 \\
\hline 27 & Indole-3-glycerol-phosphate synthase & TrpC & 4.1.1.48 \\
\hline 28 & Anthranilate phosphoribosyl transferase & TrpD & 2.4 .2 .18 \\
\hline 29 & Anthranilate synthase component I & TrpE & 4.1.3.27 \\
\hline
\end{tabular}

Table 2: The 179 non-homologous enzymes $m$. leprae

\begin{tabular}{|c|c|c|c|c|c|c|c|c|c|}
\hline Gene & EC \# & Gene & EC \# & Gene & EC \# & Gene & EC \# & Gene & EC \# \\
\hline ppgK & 2.7 .1 .63 & Adi & 4.1 .1 .19 & murG & 2.4.1.227 & & 1.2.1.- & SecA,azi,div & \\
\hline glpX & 3.1.3.11 & murI & 5.1 .1 .3 & $\operatorname{mraY}$ & 2.7.8.13 & & 3.3.2.8 & SecD & \\
\hline Fba & 4.1.2.13 & panD & 4.1.1.11 & uppP & 3.6.1.27 & & 3.1 .1 .45 & SecE & \\
\hline Fba & 1.1.1.42 & Asd & 1.2.1.11 & thiE & 2.5 .1 .3 & rpoA & & SecF & \\
\hline rpiB & 5.3.1.6 & thrB & 2.7.1.39 & thiL & 2.7.4.16 & Omega & 2.7 .7 .6 & SecG & \\
\hline otsA & 2.4.1.15 & Ask & 2.7.2.4 & ThiC & & rplO & & SPase I & 3.4 .21 .89 \\
\hline \multirow[t]{2}{*}{ otsB2 } & 3.1.3.12 & sdaA & 4.3.1.17 & ThiG & & rplR & & SPase II & 3.4.23.36 \\
\hline & 3.2.1.21 & metE & 2.1 .1 .14 & ribG & 1.1.1.193 & rpiU & & TatA & \\
\hline murA & 2.5.1.7 & ilvC & 1.1 .1 .86 & cobT & 2.4.2.21 & $\mathrm{rplV}$ & & TatB & \\
\hline murB & 1.1 .1 .158 & ilvD & 4.2 .1 .9 & $\mathrm{ribC}$ & 2.5.1.9 & rplY & & TatC & \\
\hline $\mathrm{rmlC}$ & 5.1.3.13 & Hom & 1.1 .1 .3 & ribA & 3.5 .4 .25 & $\mathrm{rpmC}$ & & YajC & \\
\hline glcB & 2.3.3.9 & dapD & 2.3 .1 .117 & ribG & 3.5.4.26 & $\mathrm{rpmD}$ & & YidC & \\
\hline $\mathrm{ppdK}$ & 2.7.9.1 & dapF & 5.1 .1 .7 & RIBB & & rpmG & & dnaE $-\alpha$ subunit & 2.7 .7 .7 \\
\hline $\mathrm{Ppc}$ & 4.1.1.31 & murF & 6.3 .2 .10 & YaaD & 4.-...- & rpmE & & dnaN $-\beta$ subunit & 2.7 .7 .7 \\
\hline gltB & 1.4.1.13 & murE & 6.3 .2 .13 & YaaE & 2.6.-.- & $\mathrm{rpmF}$ & & Subunit $-\delta$ & 2.7 .7 .7 \\
\hline $\operatorname{cynT}$ & 4.2 .1 .1 & hisD & 1.1.1.23 & nadD & 2.7.7.18 & rpmH & & DnaB & 3.6.1.- \\
\hline cysE & 2.3 .1 .30 & hisG & 2.4.2.17 & nadA & & rpmI & & DnaG & 2.7.7.- \\
\hline $\operatorname{met} X$ & 2.3.1.31 & hisI & 3.5.4.19 & COAX & 2.7.1.33 & rpmJ & & $\mathrm{rnhB}$ & 3.1 .26 .4 \\
\hline Dxr & 1.1.1.267 & hisE & 3.6.1.31 & acpS & 2.7.8.7 & Rrs & & ssb & \\
\hline pgsA & 2.7 .8 .5 & hisB & 4.2.1.19 & panC & 6.3.2.1 & Rrf & & Nfo & 3.1 .21 .2 \\
\hline pssA & 2.7.8.8 & hisA & 5.3.1.16 & panB & 2.1 .2 .11 & $\mathrm{rpsC}$ & & Tag & \\
\hline $\mathrm{Cdh}$ & 3.6.1.26 & HisF & 4.1.3.- & bioB & 2.8 .1 .6 & $\mathrm{rpsD}$ & & UVRC & \\
\hline Psd & 4.1.1.65 & HisH & 2.4.2.- & folB & 4.1 .2 .25 & rpsJ,nusE & & xseA & 3.1 .11 .6 \\
\hline dnaN & 2.7 .7 .7 & aroE & 1.1.1.25 & Folk & 2.7 .6 .3 & $\mathrm{rpsN}$ & & Dpo III & 2.7 .7 .7 \\
\hline Dgt & 3.1 .5 .1 & ML2472 & 1.3.1.12 & folP & 2.5.1.15 & $\mathrm{rpsO}$ & & PriA & 3.6.1.- \\
\hline thyX & 2.1 .1 .148 & $\operatorname{trpD}$ & 2.4.2.18 & hemD & 2.1.1.107 & $\mathrm{rps} Q$ & & RecF & \\
\hline $\mathrm{Cmk}$ & 2.7.4.14 & aroA & 2.5.1.19 & hemD & 4.2.1.75 & rpsT & & $\mathrm{RecO}$ & \\
\hline pyrH & 2.7.4.22 & aroG & 2.5 .1 .54 & COX15 & & OppB & & RecR & \\
\hline pyrF & 4.1.1.23 & aroD & 4.2.1.10 & MENC & 4.2 .1 .113 & OppC & & RuvA & \\
\hline murC & 6.3 .2 .8 & pheA & 4.2 .1 .51 & MenD & 2.5.1.64 & ProX & & RuvC & 3.1 .22 .4 \\
\hline murD & 6.3 .2 .9 & aroB & 4.2 .3 .4 & $\operatorname{argC}$ & 1.2 .1 .38 & PstA & & $\operatorname{argB}$ & 2.7.2.8 \\
\hline
\end{tabular}




\section{Bioinformation}

\begin{tabular}{|c|c|c|c|c|c|c|c|c|c|}
\hline CbiQ & & hisA & 5.3.1.24 & $\operatorname{argJ}$ & 2.3.1.1 & pstC & & MenD & 4.1.1.71 \\
\hline FhuC & 3.6 .3 .34 & OppA & & argJ & 2.3 .1 .35 & $\mathrm{RfbA}$ & & YrbD & \\
\hline FhuD & & FtsX & & YrbE & & YadH & & $\operatorname{PrrA} * * *$ & \\
\hline ddl & 6.3.2.4 & prrB & 2.7.13.3 & $\operatorname{ctaA}$ & & $\operatorname{trpC}$ & 4.1.1.48 & & 2.7.1.- \\
\hline Alr & 5.1.1.1 & $\operatorname{trp} \mathrm{A}$ & 4.2.1.20 & mprB & 2.7 .13 .3 & & 1.2.-.- & & \\
\hline
\end{tabular}

Table 3: Essential enzymes of $M$. leprae

\begin{tabular}{|c|c|c|c|}
\hline S. No & $\begin{array}{c}\text { Enzyme } \\
\text { name }\end{array}$ & Name of the Enzyme & Name of the pathway \\
\hline \multirow[t]{2}{*}{1} & murC & UDP-N-acetylmuramate-L-alanine ligase & $\begin{array}{l}\text { D-Glutamine \& D-Glutamate } \\
\text { metabolism. }\end{array}$ \\
\hline & & & Peptidoglycan biosynthesis \\
\hline \multirow[t]{2}{*}{2} & murD & UDP-N-acetylmuramoyl-L-alanyl-D-glutamate synthetase & $\begin{array}{l}\text { D-Glutamine \& D-Glutamate } \\
\text { metabolism. }\end{array}$ \\
\hline & & & Peptidoglycan biosynthesis \\
\hline \multirow[t]{2}{*}{3} & murE & UDP-N-acetylmuramoylalanyl-D-glutamate--2,6-diaminopimelate & Peptidoglycan biosynthesis \\
\hline & & ligase & Lysine biosynthesis \\
\hline \multirow[t]{2}{*}{4} & murF & UDP-N-acetylmuramoylalanyl-D-glutamyl-2,6-diaminopimelate--D- & Peptidoglycan biosynthesis \\
\hline & & alanyl-D-alanyl ligase (D-alanine:D-alanine-adding enzyme) & Lysine biosynthesis \\
\hline 5 & murG & $\mathrm{N}$-acetylglucosaminyl transferase & Peptidoglycan biosynthesis \\
\hline 6 & mraY & Phospho-N-acetylmuramoyl-pentapeptide-transferase & Peptidoglycan biosynthesis \\
\hline 7 & rmlC & Putative dTDP-4-dehydrorhamnose 3,5-epimerase & Nucleotide sugars metabolism. \\
\hline & & & Polyketide sugar unit biosynthesis \\
\hline \multirow[t]{2}{*}{8} & alr & Alanine racemase & D-Alanine metabolism. \\
\hline & & & Alanine \& Aspartate metabolism \\
\hline
\end{tabular}

